\title{
The April 2020 Issue
}

SIGCOMM Computer Communication Review (CCR) is produced by a group of members of our community that spend time to prepare the newsletter that you read every quarter. Olivier Bonaventure served as editor during the last four years and his term is now over. It is my pleasure to now serve the community as the editor of CCR. As Olivier and other editors in the past did, we'll probably adjust the newsleter to the evolving needs of the community. A first change is the introduction of a new Education series led by Matthew Caesar, our new SIGCOMM Education Director. This series will be part of every issue of CCR, and will contain different types of contributions, not only technical papers as in the current issue, but also position papers (that promote discussion through a defensible opinion on a topic), studies (describing research questions, methods, and results), experience reports (that describe an approach with a reflection on why it did/did not work), and approach reports (that describe a technical approach with enough detail for adoption by others).

This April 2020 issue contains five technical papers, the first paper of our new education series, as well as three editorial notes.

The first technical paper, RIPE IPmap Active Geolocation: Mechanism and Performance Evaluation, by Ben Du and his colleagues, introduces the research community to the IPmap single-radius engine and evaluates its effectiveness against commercial geolocation databases.

It is often believes that traffic engineering changes are rather infrequent. In the second paper, Path Persistence in the Cloud: A Study of the Effects of Inter-Region Traffic Engineering in a Large Cloud Provider's Network, Waleed Reda and his colleagues reveal the high frequency of traffic engineering activity within a large cloud provider's network.

In the third paper, The Web is Still Small
After More Than a Decade, Nguyen Phong Hoang and his colleagues revisit some of the decade-old studies on web presence and colocation.

The fourth paper, a repeatable paper originated in the IMC reproducibility track, An Artifact Evaluation of NDP, by Noa Zilberman, provides an analysis of NDP (New Data centre protocol). NDP was first presented at ACM SIGCOMM 2017 (best paper award) and proposes a novel data centre transport architecture. In this paper, the author builds the analysis of the artefact proposed by the original authors of NDP, showing how it is possible to carry out research and build new results on previous work done by other fellow researchers.

The Low Latency, Low Loss, Scalable throughput (L4S) architecture addresses this problem by combining scalable congestion control such as DCTCP and TCP Prague with early congestion signaling from the network. In our fifth technical paper, Validating the Sharing Behavior and Latency Characteristics of the L4S Architecture, Dejene Boru Oljira and his colleagues validate some of the experimental result(s) reported in the previous works that demonstrate the co-existence of scalable and classic congestion controls and its low-latency service.

The sixth paper, also our very first paper in the new education series, An Open Platform to Teach How the Internet Practically Works, by Thomas Holterbach and his colleagues, describes a software infrastructure that can be used to teach about how the Internet works. The platform presented by the authors aims to be a much smaller, yet representative copy of the Internet. The paper's description and evaluation is focused on technical aspects of the design, but as a teaching tool it may be more helpful to describe more about pedagogical issues.

Then, we have three very different editorial notes. The first, Workshop on In- 
ternet Economics (WIE 2019) report, by kc Klaffy and David Clark, reports on the 2019 interdisciplinary Workshop on Internet Economics (WIE). The second, strongly related to the fourth technical paper, deals with reproducibility. In Thoughts about Artifact Badging, Noa Zilberman and Andrew Moore illustrate that the current badging scheme may not identify limitations of architecture, implementation, or evaluation. Our last editorial note is a comment on a past editorial, "Datacenter Congestion Control: Iden- tifying what is essential and making it practical" by Aisha Mushtaq, et al., from our July 2019 issue. This comment, authored by James Roberts, disputes that shortest remaining processing time (SRPT) scheduling is the crucial factor in achieving good flow completion time (FCT) performance in datacenter networks.
Steve Uhlig

CCR Editor 